\title{
Classifying Features Persuading the Use of Long Lasting Insecticide Treated Nets (LLINs) and Its Implications in Exterminating the Incidence of Malaria-Death in Ghana
}

\author{
Anthony Joe Turkson \\ Department of Mathematics, Statistics and Actuarial Sciences, Takoradi Technical University, Ghana \\ e-mail: anthony.turkson@ttu.edu.gh
}

\begin{abstract}
This is a cross-sectional quantitative study purported to identify features deemed to persuade the usage of LLINs in exterminating incidences of malaria-death in Ghana. The population consisted of mothers and caregivers of children under five in Asamankese a district in the Eastern region of Ghana. Questionnaires were developed based on the profile and the set of study objectives, it sought information on socio-economic variables, knowledge level on LLINs, and influence of climatic and environmental factors on LLINs usage. Data were coded and keyed into SPSS version 20. Frequencies, percentages, means, standard deviations, graphs and tables were used to explore the data. Chi-square test was used to do further investigation.
\end{abstract}

It was revealed that LLINs usage was influenced by a group of features including: background characteristics of household; socio-economic variables, environmental variables and knowledge on importance of LLINs. There was an association between LLINs usage and monthly income of caregivers $(p<0.05)$. Furthermore, there was a significant relationship $(p<0.05)$ between environmental features and LLINs usage. There was a relationship $(p<0.05)$ between one's knowledge and use of LLINs. In addition, there was a relationship between usage and the number of times per month visits were made to the hospitals for health care. Environmental factors permitted the use of LLINs, Eighty-six $(86 \%)$ of the respondents who used LLINs did use it because the weather aided them.

It is recommended that behavior change education be intensified in the region so that more people can accept and adopt a lifestyle that will protect them from the deadly

Received: October 22, 2021; Accepted: November 29, 2021

2020 Mathematics Subject Classification: 62H17.

Keywords and phrases: Chi-square, LLINs, malaria, risk factors, children under five. 
malaria diseases. Efforts must be made by the major players in the health sector to make the net readily available in the communities at low prices to enable the ordinary Ghanaian to purchase it.

\section{Introduction}

Malaria remains a major challenge in sub-Saharan Africa and Ghana is not an exception. Effective malaria transmission control requires evidence-based targeting and utilization of resources. The recent world malaria report estimated that 216 million cases of malaria and 445,000 deaths occurred worldwide in 2016; the number of cases increased by approximately 5 million compared to the previous year [1]. Malaria burden is greatest in sub-Saharan Africa (SSA) where an estimated $90 \%$ of all malaria deaths occur, and children under 5 years old account for $78 \%$ of all deaths [2]. The World Health Organization (WHO) estimated that one child in SSA dies from malaria every 2 minutes [3]. The disease is caused by a plasmodium parasite. These parasites are transmitted from human to human via Anopheles mosquitoes, most commonly Anopheles Gambia in Africa. Plasmodium falciparum, is found worldwide in tropical and subtropical areas.

Initial symptoms of malaria include fever, headache, and vomiting. In more severe cases of malaria, such as those caused by Plasmodium falciparum, coma and death are common if untreated. Even when treatment is administered, impaired development in children is common after severe malaria. According to the latest World malaria report, released on 30 November 2020, there were 229 million cases of malaria in 2019 compared to 228 million cases in 2018. The estimated number of malaria deaths stood at 409000 in 2019, compared with 411000 deaths in 2018. In 2019, nearly half of the world's population was at risk of malaria [4].

Risk factors pertaining to malaria prevalence considered in the current study are area of residence (urban/rural), age of the child in months, use or non-use of long-lasting insecticide treated nets (LLINs), household wealth status and mother's education level. The behavioural risk for malaria in the Machodinho resettlement area in the Amazonian forests of Brazil was examined [5]. Analysis of the study suggested that economic status and knowledge of the importance and behavoiur of the mosquito in transmitting malaria were significant factors in determining prevalence risk irrespective of whether preventive precautions, like, dichlorodiphenyl trichloroethane (DDT) spraying of houses and cleaning of vector breeding sites were undertaken in the endemic areas. The researchers found out that a higher economic status combined with better knowledge of the vector 
and DDT spraying of houses decreased the risk of infection. They suggested a more enhanced control programmes on behalf of poorer people especially migrants in order to diminish the disease burden for them.

The association between area of residence and malaria prevalence is well known. Living in rural areas was positively associated with the probability of a child having a positive malaria outcome. Similar results were reported by [6] who analyzed and reported data from the 2008 Ghana Demographic Health Survey. Malaria risk in urban areas is known to differ from those in rural area. Malaria is often referred to as a disease of poverty [7].

A study by [8] on malaria in the Greater Accra Region of Ghana revealed that factors that were perceived as causing malaria were malnutrition, mosquitoes, excessive heat, excessive drinking, flies, fatigue, dirty surroundings, unsafe water, bad air and poor hygiene. Almost all the adolescents at that time had no idea how the disease was spread from person to person, while the symptoms of clinical malaria were also frequently considered to be yellowish eyeball, chills and shivering, headache, a bitter taste, body weakness and yellowish urine, the study added.

The disease can be diagnosed by a microscopic examination or use of Rapid Diagnostic Test kits (RDTs) of the patient's blood and can normally be cured by taking anti-malarial drugs. However, there are some regions where parasites have developed resistance to these drugs. Key interventions to control malaria include: prompt and effective treatment with artemisinin-based combination therapies; use of insecticidal nets by people at risk; and indoor residual spraying with insecticide to control the vector mosquitoes [9].

\subsection{The long lasting insecticide treated nets (LLINs)}

All mosquito nets act as a physical barrier, preventing access to humans from mosquito bites and thus protection against malaria to the individual using the net. Pyrethroid insecticides, which are used to treat nets, have an excito-repellent effect that adds a chemical barrier to the physical one, reducing human-vector contact and increasing the protective efficacy of the mosquito nets. Most commonly, the insecticide kills the malaria vectors that come into contact with the LLINs. By reducing the vector population in this way, LLINs provide protection for all people in the community, including those who do not sleep under nets, this means that, the vector is either killed or rendered inactive to transmit parasite from one protected person to a non-protected person. 
A number of studies have found that LLINs provide varying degrees of protection against malaria morbidity, anemia and low birth weight. In a trial of untreated bed nets in the Gambia, the nets were found to reduce the number of infective bites but not enough to reduce morbidity from malaria. [10] noted that there had been a robust evidence of the efficacy of LLINs in reducing the prevalence malaria parasite, incidence, and all-cause child mortality. A study conducted in Ethiopia with the aim of examining the factors associated with the use and non-use of LLINs revealed that out of the 857 households surveyed, 91 percent of them owned at least one LLINs [11]. LLINs distribution has a strong track record of significantly reducing mortality in both repeated, randomized controlled trials and in larger-scale, country-level distribution efforts. [12] had investigated the effect of LLINs on two genes in a large community-based LLINs trial site in western Kenya. They noted that the high diversity in these two genes had been maintained overtime despite marked reductions in malaria transmission due to LLINs use. Manufacturers delivered about 253 million LLINs to malaria endemic countries in 2019, an increase of 56 million LLINs compared with 2018. About $84 \%$ of these LLINs were delivered to countries in sub-Saharan Africa.

By 2019, 68\% of households in sub-Saharan Africa had at least one LLINs, increasing from about $5 \%$ in 2000. Subsequently, the percentage of the population sleeping under an LLINs also increased considerably between 2000 and 2019, for the whole population (from $2 \%$ to $46 \%$ ), for children aged under 5 years (from $3 \%$ to $52 \%$ ), and for pregnant women (from $3 \%$ to $52 \%$ ) [13].

In a national cross-sectional survey, it came to light that there was some degree of association between LLINs-usage and malaria prevention. There was a 23 per cent reduction in all-cause child mortality [10]. In response to the increased global prioritization of malaria prevention and treatment, attention has now been shifted from prevention campaigns to monitoring and evaluation (M\&E) of the interventions, in this direction, many countries are currently using large multistage cluster sample surveys to monitor malaria outcome indicators on a regional and national level [14]. Attention is now shifted to some of the popular features deemed to have some effects on LLINs usage.

\subsection{Knowledge on LLINs and usage}

Several studies have shown that poor perception and knowledge on malaria or its control is common among people living in areas where malaria is endemic. While misconception about the cause and prevention of malaria was significantly associated 
with non-use of LLINs, no association between knowledge of symptoms and risk of malaria and the use of LLINs were observed. A study conducted by [15] with the view of evaluating the knowledge, attitude and practice (KAP) about LLINs at household level in rural sub-distracts of Kolla Tembien and its usage revealed that the overall knowledge, attitude and practice of LLINs usage was satisfactory in the study district. Knowledge on personal illness control measures, personal preventive measures and medical treatment schemes were also identified.

[16] reported that the LLINs used to protect people against mosquito bites had proved to be highly and cost-effective intervention scheme. In supporting their assertion, they noted that there were evidences from the public health that the prevalence of Plasmodium falciparum infection in community-based randomized controlled trials (RCT) in endemic regions had shown average reductions of $20 \%$ in all causes of mortality in children under 5 years in the space of two years of increasing LLINs usage from zero to 50-70\%. The use of ITNs is largely affected by the knowledge of people. Behavioral patterns of people-utilization of the ITN are dependent on their knowledge on the consequence of nonuse. Researchers give varied indications on the use of the LLINs and peoples level of knowledge. It has been alluded that despite available evidences that the use of LLINs decreases malaria-related morbidity and mortality, its use in SSA remains relatively low.

\subsection{Climatic and environmental factors and LLINs usage}

A study aimed at ascertaining the linkages between exposure to the environment and health hazards revealed that the hazard ratio of the exposed group was higher than the less exposed group. The study also revealed that dwellers who subscribed to LLINs as means of reducing exposure to the malaria parasite had better and longer survival prognosis than non-subscribers $[17,18]$ observed that after a decade of investment in malaria control across sub-Saharan Africa, there was the need to evaluate the impact of the investment on reducing the environmental burden on households. Environmental factors include but not limited to contamination of the following: air; food; water; fingers; skin; soil; inanimate objects; insect vectors; and bushy dwelling place. Climatic condition may influence mosquito breeding and hence transmission of malaria. Anopheles gambiae breed in temporary pools such as roadside ditches and drains, burrow pits, hoof prints, wheel nuts, and puddles and highly depend on the rains for their numbers. The rainy season actually favours the prolific breeding in the sites, which in fact are numerous. These species have the highest population density during the rainy season and these accounts for the high incidence of malaria at this period of the year. In 
the hot dry seasons, substantial number of people may sleep outside in the open air, as a consequence of being bitten more frequently by species of mosquitoes. During the wet and cold season, the species multiply rapidly thus increase their population and biting frequency.

\subsection{Socio economic factors and LLINs usage}

Some identified socio-economic maternal factors include age, parity, and birth interval. Others include: occupation; life style; and domestic activities. Occupation of the people such as farming, fishing, animal rearing, stone quarrying, and petty trading, have an impact on the transmission of the disease due to the exposure to the bite of the infective species. The life style of the people such as sleeping outside, watching television or visiting the video centre, cinema centre, and drinking bars get them exposed to the species. Domestic activities of the people refer to situations where, drying of farm produce, cooking and washing are normally done outside the rooms.[19] noted that the prevalence of malaria for households with clean water was lower than households without clean water, it was also noted that diagnosis of malaria was higher for people living in thatch and mud houses and earth dung plaster floor than people living in the other types of houses. [20] reported that efforts by health workers, volunteers and the media in relating LLINs messages to the public was very useful in increasing use of bed nets. [21] also noted that the effective control of malaria could enhance the attainment of international malaria targets by 2015. [22] intimated that even though long-lasting insecticidal nets were effective materials for malaria prevention, nevertheless, national level campaigns in several countries had often run out of nets in the course of the distribution, they noted that this situation reveals a problem in assessing the actual quantity needed for distribution [23, 25].

This study sought to investigate the features of knowledge, environment and socioeconomic variables and their impact on LLINs usage in children under five years. The following hypotheses were tested:

$\mathbf{H}_{\mathbf{0}}$ : There is no association between knowledge of mothers and caregivers on and use of LLINs among children under five years.

$\mathbf{H}_{\mathbf{0}}$ : Use of LLINs in children under five years is not linked to the environmental set up of mothers or caregivers.

$\mathbf{H}_{0}$ : Use of LLINs in children under five years is not related to socioeconomicparameters of mothers and caregivers. 


\section{Materials and Methods}

Cross-sectional quantitative design was adopted for this study. This design was used to unearth the significant features that were deemed to affect the use of LLINs in malaria control in children under five in Asamankese a district in the Easterm region of Ghana. This design was used because it is relatively quicker, easier and good for studies of this nature. It also provides data on health related characteristics in a defined population at a given point in time. The study population consisted of mothers and caregivers of children under five in the Eastern region of Ghana. The area chosen for the studywas made up of a combination of urban and rural dwellers whose dominant business was trading and subsistence farming. Apart from the members of the community who were involved in 'white collar jobs', the income of the indigenes tend to be unstable due to the seasonality of their occupation. The climate in the area is typically wet equatorial with the major rainy season running from late February to early July and minor from mid-September to early November. The dry season lasts for four months; December to April with its peak in February. The prevalence of appropriate LLINs use in the study area was not known, thus a prevalence figure of $50 \%$ was assumed and used to calculate the sample size. With $95 \%$ confidence level, the sample size was 384.16 and was approximated to 500 (adjusting for $96 \%$ response rate. This was also done to cater for errors and sample variability in the study population. The sample size was estimated using the formula:

$$
n \geq \frac{Z^{2} p(1-p)}{d^{2}}
$$

where:

$Z=1.96$

$p=$ Proportion of the sample that is assumed to be using LLINs

$d=$ Error margin. (The margin of error one is prepared to make determines the size of sample he will take and vice versa, it is statistically pegged at 5\%)

$$
n=\frac{1.96 \times 0.5 \times 0.5}{0.05^{2}}=384.2[24] .
$$

The questionnaire was developed based on the profile and the set of study objectives. The questionnaire sought information on socio-economic variables, knowledge level on the importance of LLINs vis a vis its usage and the influence of environmental factors on LLINs usage. The questionnaire was reviewed by fellow researchers from the public health sector and pretested on a small sample to ensure content validity. Trained 
enumerators assisted in the enumeration of respondents over a 3-day period. The participants were selected using the snowball technique, where respondent mothers and caregivers of children under five gave referrals to the research team; this then led to other mothers and caregivers.

The data was analyzed using the Statistical Package for Social Sciences (SPSS) version 20. It was coded and keyed into the software. Frequencies, percentages, mean, standard deviation, graphs and tables were used to explore the data. Chi-square was used to further analyze the data. The significance of the result was pegged at probability value $(p<0.05)$.

\section{Results}

A total of 500 households were investigated during the study. The demographic data is presented in Table 1 . Of these, $67.8 \%$ were women while the remaining $32.2 \%$ were men, indicating that women were typically the custodians of children under 5 years.

The ages of the respondents ranged from 15 to 65 years with a median age of 30 years and standard deviation 8.417. The age group with the largest number of participants was the 15-29 year group which constituted $47 \%$; this was followed by the age group 3044 years which constituted (45.8\%).

About $92 \%$ of the respondents were gainfully employed. These respondents were either artisans or engaged in farming, trading and other activities. The table further shows that only $8.8 \%$ of the respondents had no formal education. Over $50 \%$ of respondents had junior high education, while the remaining 39\% was distributed among those who had primary $(19.4 \%)$, senior high $(11.8 \%)$ and tertiary (7.4\%) education.

From Table 2, we could see that 368 married couples (73.6\%), eighty eight singles $(17.6 \%)$ and forty four $(8.8 \%)$ either divorced or separated were included in the study. 
Table 1. Socio-demographic profile of respondents.

\begin{tabular}{lcc}
\hline Variables & Frequency & Percentage \\
\hline Gender & & \\
Male & 161 & 32.2 \\
Female & 339 & 67.8 \\
Age & & \\
$15-29$ & 235 & 47.0 \\
$30-44$ & 229 & 45.8 \\
$45-59$ & 30 & 6.0 \\
60 and above & 6 & 1.2
\end{tabular}

\section{Employment status}

Employed $\quad 462 \quad 92.4$

\section{Unemployed}

Occupation

38

7.60

Artisan

149

32.25

Farming

70

15.15

Trader

201

43.51

\section{Other}

Level of Education

42

9.09

Primary

97

19.40

Junior high or MSLC 263

52.60

Senior high

Tertiary

No formal education 44

8.80

Source: Field work 
Table 2. Background characteristics of mothers and care givers.

\begin{tabular}{lrr} 
Household variables & Frequency & Percentage \\
\hline Marital status & & \\
$\quad$ Single & 88 & 17.6 \\
Married & 368 & 73.6 \\
$\quad$ Divorced & 27 & 5.4 \\
Separated & 17 & 3.4 \\
Number of children & & \\
1 & 145 & 29.0 \\
2 & 151 & 30.2 \\
3 & 135 & 27.0 \\
4 & 59 & 11.8 \\
5 & 5 & 1.0 \\
6 & 4 & 0.8 \\
7 & 1 & 0.2
\end{tabular}

Number of children aged between 0 and 5 years

\begin{tabular}{rrr}
1 & 317 & 63.4 \\
2 & 175 & 35.0 \\
3 & 7 & 1.4 \\
4 & 1 & 0.2 \\
\hline
\end{tabular}

Source: Field work 
Table 3. Baseline characteristics of households.

\begin{tabular}{lrl}
\hline Explanatory variables & Frequency & Percentage \\
\hline Do you own ITNs? & & \\
Yes & 431 & 86.2 \\
No & 69 & 13.8 \\
Number of ITNs in households & & \\
1 & 149 & 34.57 \\
2 & 179 & 41.53 \\
3 & 83 & 19.26 \\
4 & 20 & 4.64
\end{tabular}

Mean: 1.67 , standard deviation: 1.037

Where did you acquire ITNs from?

ITNs hang-up campaign 358

83.06

Health facility

$69 \quad 16.01$

Other (friends, church)

$4 \quad 0.93$

Number of children under 5 who slept in ITN last night

$\begin{array}{lrr}0 \text { (years) } & 31 & 7.19 \\ 1 & 258 & 59.86 \\ 2 & 137 & 31.79 \\ 3 & 5 & 1.16\end{array}$

Were ITN observed hanging?

Yes

$355 \quad 82.37$

No

76

17.63

Source: Field work $\mathrm{N}=500$ 
From Table 2, we could infer that about $63.4 \%$ of respondents had a child under five years, $35 \%$ had two children under five years while $1.6 \%$ had three or four children under five years. The result also shows that the highest number of children a family could have was seven, which was just $0.2 \%$ of the respondents. The majority of them had between one to three children. The average number of child per household was two.

With an average of two LLINs per household, 431 (86.2\%) out of the 500 respondents owned LLINs. About $83 \%$ of those who owned LLINs acquired it from the free LLINs door-to-door hang-up campaign. Those who acquired LLINs from health facilities and other sources, formed 16 and 1\% respectively. About 82\% of LLINs were observed hanging during the study. More than $92 \%$ of children under five years slept in LLINs the night before the interview (Table 3).

Figure 1 represents a multi-response variable, from it, we could see that $79.4 \%$ of respondents believed that the use of LLINs would reduce the burden of malaria on their under five children, $44 \%$ were of the view that children slept better in LLINs. Over 33\% of respondents thought that it would help save money for other purposes. Finally, 36.6\% believed that the use of LLINs saved the time one would spend visiting health facilities or hospitals. From Table 4, 465/500 respondents (93\%) knew where to acquire LLINs. A little above $64 \%$ of respondents mentioned the health facilities as the source of acquiring LLINs, while $26 \%$ acquired it from the chemical or pharmacy shops, only $8.4 \%$ mentioned that they got the nets from other sources like gifts and donations. $10.8 \%$ of the mothers and care givers of children under five did not know how to hang the net. More than $71 \%$ indicated that the hanging process was easy. Finally, $97.6 \%$ of respondents affirmed that they knew how to use the LLINs.

Table 5 shows the relationship between background characteristics and LLINs ownership and usage. From the table, we could see that there was a significant relation between LLINs ownership and educational level $p<0.05$. The results further revealed that ownership was more among those with lower levels of education (primary and JHS) than those with higher levels. In addition, there was a significant relation between LLINs usage and the educational level of mothers and caregivers of children under 5years. There was a slight decreasing trend in the level of education and use of LLINs, starting from SHS to the primary level as shown in Table 6. 
Table 4. Respondents knowledge on use of ILLNs and where to acquire them.

Question $\quad$ Frequency $\quad$ Percentage

Do you know where to acquire LLINs?

Yes

465

93.0

No

35

7.0

Where to acquire LLINs

Health facility

324

64.8

Chemical Shop/pharmacy

134

26.8

Other

42

8.4

Do you know how to hang LLINs?

Yes

446

89.2

No

10.8

Process of hanging LLINs

Easy

71.8

Difficult

Do you know how to use LLINs?

Yes

No

2.4

Source: Field work, $\mathrm{N}=500$

Table 5. Relationship between background characteristics and LLINs ownership and use.

\begin{tabular}{llllll}
\hline Ownership of ITN & Yes & No & Total & Chi-square & P-value \\
\hline
\end{tabular}

Level of education

Primary

$\begin{array}{rrr}82 & 15 & 97 \\ 218 & 45 & 263\end{array}$

Senior high

$58 \quad 1 \quad 59$

12.109

0.017

Tertiary

35

2

No formal education 
ITN use among caregivers of children under 5years

Level of education

Primary

$82 \quad 15 \quad 97$

Junior high or MSLC

$212 \quad 51 \quad 263$

Senior high

58

$1 \quad 59$

32.791

0.000

Tertiary

$\begin{array}{lll}35 & 2 & 37\end{array}$

No formal education

$32 \quad 12$

44

\section{ITN usage among children under 5 years}

\section{Level of education}

Primary

Junior high or MSLC

Senior high

Tertiary

No formal education
80

105

47

36

39

$17 \quad 97$

$63 \quad 168$

15.162

0.004

Source: Field study.

Table 6. Relationship between knowledge, environmental factors and ownership of LLINs on usage.

Yes No Total Chi-square P-value

\section{ITN usage among children under 5 years}

Knowledge on ITN Use

$\begin{array}{lrrrrr}\text { Yes } & 410 & 71 & 481 & 19.31 & 0.000 \\ \text { No } & 9 & 10 & 19 & & \end{array}$

Does the environment permit use of ITN?

$\begin{array}{lrrrrr}\text { Yes } & 350 & 74 & 424 & 11.311 & 0.001 \\ \text { No } & 50 & 26 & 76 & & \end{array}$

\section{ITN ownership}

$\begin{array}{lrrrrr}\text { Yes } & 419 & 12 & 431 & 414.067 & 0.000 \\ \text { No } & 0 & 69 & 69 & & \end{array}$

Source: Field study 
Table 7. Tests of association between predetermined factors and use of ITN.

Use of ITN Yes No Chi-Square P-value

Knowledge of how to use ITN

$\begin{array}{lrrrr}\text { Yes } & 388 & 58 & 31.620 & <0.001 \\ \text { No } & 31 & 23 & & \end{array}$

Effect of ITN use on visit to source health care services

$\begin{array}{lrrrr}\text { Same } & 58 & 371 & 44.984 & <0.001 \\ \text { Reduced } & 355 & 23 & & \\ \text { Increased } & 5 & 8 & & \\ \text { Other } & 1 & 13 & & \end{array}$

Environmental factors affecting use of ITN

$\begin{array}{lrrrr}\text { No } & 55 & 2 & 18.627 & 0.003 \\ \text { Yes } & 364 & 60 & & \end{array}$

Knowledge (heard, seen or read anything) of ITN

$\begin{array}{lrrrr}\text { Yes } & 410 & 71 & 19.310 & <0.001 \\ \text { No } & 9 & 10 & & \end{array}$

Those who owned and used LLINs also believed that children were at risk of getting malaria if they did not sleep under LLINs.

There was a significant relationship $(p<0.05)$ between LLINs ownership and usage. There was also a significant relationship between employment status parents and the use of LLINs among children under five $(p=0.007<0.05)$. There was also an association between LLINs use and the monthly income of respondents $(p<0.05)$. Table 6 revealed that there was a significant relationship $(p<0.05)$ between environmental factors and LLINs usage. The significant test (Table 7) also revealed that there was a relationship $(p<0.01)$ between one's knowledge and use of LLINs in addition there was a relationship between usage and the number of times per month they visited hospitals to source for health care. As to whether the environmental factors permitted the use of LLINs, it was found out that it did influence its use; it was revealed that $86 \%$ of the respondents who used LLINs did use it because the weather aided them. There was a significant relationship ( $p<0.05)$ between the climatic condition (environmental factor) and use of LLINs. Finally, respondents' knowledge on LLINs had a significant influence $(p<0.05)$ on their usage (Table 7). From Figure 2, we could see that $75 \%$ of respondents had a 
reduction in the frequency of visits to health facilities as a result of use of LLINs. 19\% did not observe any change in their visit to the health facility.

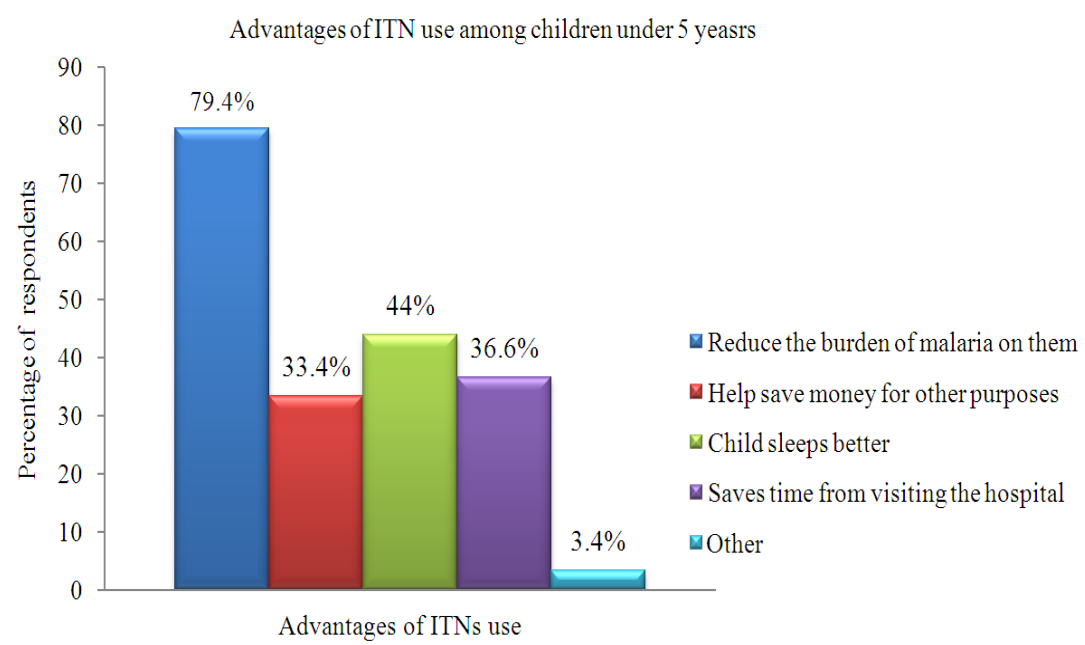

Figure 1. Histogram displaying the knowledge of the advantages of ITNs usage among children under 5 years old as revealed in the study.

\section{Use of LLINs and frequency of visit to health facility}
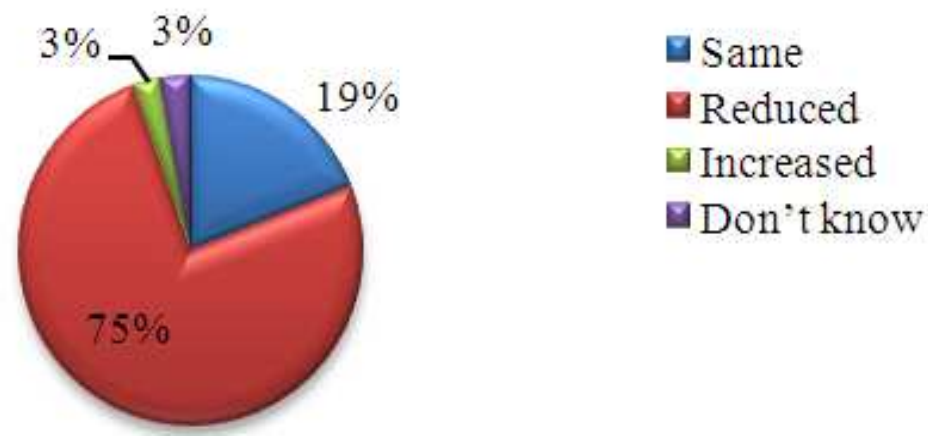

Figure 2. Pie chart showing effect of LLINs use and the frequency of visits to health facilities.

\section{Discussion and Conclusion}

The target population was children under five. 500 mothers and caregivers were interviewed. The results showed that $68 \%$ of the participants were women. This explains how involved women were when it comes to taking care of children. Nonetheless, there was an increasing involvement of men in the upbringing of children as compared to 
previous surveys. As much as $93 \%$ of the participants were youths while $74 \%$ were married. Again, $92 \%$ of the participants were gainfully employed. In all, $72 \%$ of the participants had at least the basic education with $7 \%$ having tertiary education. There was a fluctuating trend in the level of education and LLINs ownership and usage with JHS graduates having the highest usage of $51 \%$.

The caregivers and mothers had knowledge on the nets and its usage. As much as $82 \%$ of them owned and used LLINs.

It was also found out that $86 \%$ of the participants owned LLINs but only $82 \%$ used them regularly. Eighty-three percent $(83 \%)$ of those who owned LLINs acquired them from the LLINs door-to-door distribution and hang-up campaign. The study revealed that majority of those who had knowledge on importance of LLINs used it more.

It was realized that $59 \%$ of the participants were engaged in either trading or farming which did not give enough income for them and their families. The results showed that people with lower income levels tend to own and use LLINs than those with higher incomes.

Though the incomes of the participants were not high compared to the standard of living now, they were willing to buy the nets and let their children sleep in them because of the benefits that can be derived from it. However, people who were using the LLINs were willing to buy it than those who were not sleeping in them. Some schools of thought were of the view that the nets were inexpensive considering their benefits and their efficacy to avert childhood deaths particularly in sub-Saharan Africa. The multivariate analysis indicated that income levels were determinant to LLINs use. However, the higher the expected monthly salary, the less likely one was to use LLINs.

In the tropics, the weather could be a discouragement to the appropriate use of LLINs. The structures in Africa and in peri-urban areas for that matter do not match up to the urban settings. The outcome therefore was that, there was less likelihood of one using LLINs with a smaller room space.

LLINs use in the study area was influenced by a group of factors which included the following: Background characteristics of household, socio-economic status, environmental factors and knowledge on importance of LLINs.

There was a significant relationship between LLINs ownership and usage. There was also a significant relationship between employment status and the use of LLINs among children under five. Moreover, there was an association between LLINs use and the 
monthly income of respondents. There was again a significant relationship between environmental factors and LLINs usage. One's knowledge on and use of LLINs were also associated.

It is recommended that behavior change education should be intensified in the municipality so that more people can accept and adopt a lifestyle that will protect them from the deadly malaria diseases. While advocating continuation of education on the use of the LLINs, it is recommended that efforts must be seriously made by the major players in the health sector to make the net readily available in the communities at low prices to enable the ordinary Ghanaian to purchase it.

\section{Ethical Consideration}

Permission was sought at all levels; from the municipal health directorate and submunicipal offices. Letters were sent to and approved by these offices to ask for their consent and permission to conduct the study in the area. The participants consented to be part of the study. The researchers explained in the local dialect the content of the research for the benefit of those who could not read. Each of the participants was visited in their home.

\section{References}

[1] World malaria report 2017, Geneva: World Health Organization, 2017. https://www.who.int/malaria/publications/world-malaria-report-2017/en/

[2] R. Yankson, E. A. Anto and M. G. Chipeta, Geostatistical analysis and mapping of malaria risk in children under 5 using point-referenced prevalence data in Ghana, Malar. J. 18 (2019), 67. https://doi.org/10.1186/s12936-019-2709-y

[3] World malaria report 2015, Geneva: World Health Organization, 2015. http://www.who.int/iris/handle/10665/200018

[4] Centres for Disease Control and Prevention, Malaria Worldwide, 2014. http://www.cdc.gov/malaria/malaria_worldwide/

[5] R. E. Fernandez Castilla and D. O. Sawyer, Malaria rates and fate: A socioeconomic study of malaria in Brazil, Social Sciences \& Medical 37 (1993), 1137-1145. https://doi.org/10.1016/0277-9536(93)90253-Z

[6] S. H. Nyarko and A. Cobblah, Sociodemographic determinants of malaria among underfive children in Ghana, Malar. Res. Treat. 2014 (2014), Art. ID 304361, 6 pp. https://doi.org/10.1155/2014/304361 
[7] B. S. C. Uzochukwu and O. E. Onwujekwe, Socio-economic differences and health seeking behaviour for the diagnosis and treatment of malaria: a case study of four local government areas operating the Bamako initiative programme in south-east Nigeria, International Journal for Equity in Health 3 (2004), 6.

https://doi.org/10.1186/1475-9276-3-6

[8] W. K. Asenso-Okyere, Socioeconomic factors in malaria control, World Health Forum 15 (1994), 265-268.

[9] WHO, Malaria, 2014. http://www.who.int/topic/malaria/en/

[10] T. P. Eisele and R. W. Steketee, African malaria control programs deliver ITNs and achieve what the clinical trials predicted, PLoS Med. 8(9) (2011), e1001088. https://doi.org/10.1371/journal.pmed.1001088

[11] C. A. Baume, R. Reithinger and S. Woldehanna, Factors associated with use and non-use of mosquito nets owned in Oromia and Amhara Regional States, Ethiopia, Malar. J. 8 (2009), 264. https://doi.org/10.1186/1475-2875-8-264

[12] GiveWell, Program: distribution of insecticide-treated nets (ITNs) to prevent malaria 2009 Report. https://www.givewell.org/international/technical/programs/insecticidetreated-nets/2009-report

[13] S. K. Kariuki, J. Njunge, A. Muia, G. Muluvi, W. Gatei, F. ter Kuile, D. J. Terlouw, W. A. Hawley, P. A. Phillips-Howard, B. L. Nahlen, K. A. Lindblade, M. J. Hamel, L. Slutsker and Y. P. Shi, Effect of malaria transmission reduction by insecticide-treated bed nets (ITNs) on the genetic diversity of Plasmodium falciparum merozoite surface protein (MSP-1) and circumsporozoite (CSP) in western Kenya, Malar. J. 12 (2013), 295. https://doi.org/10.1186/1475-2875-12-295

[14] C. Biedron, M. Pagano, B. L. Hedt, A. Kilian, A. Ratcliffe, S. Mabunda and J. J. Valadez, An assessment of Lot Quality Assurance Sampling to evaluate malaria outcome indicators: extending malaria indicator surveys, Int. J. Epidemiol. 39 (2010), 72-79. https://doi.org/10.1093/ije/dyp363

[15] Z. Tomass, T. Dejene and D. Kidane, Knowledge, attitude and practice (KAP) about insecticide treated net (ITN) usage against malaria in Kolla Tembien district, Tigray, Ethiopia, Momona Ethiopian Journal of Science 3 (2011), 64-77.

https://doi.org/10.4314/mejs.v3i2.67713

[16] H. E. Atieli, G. Zhou, Y. Afrane, M.-C. Lee, I. Mwanzo, A. K. Githeko and G. Yan, Insecticide-treated net (ITN) ownership, usage, and malaria transmission in the highlands of western Kenya, Parasites Vectors 4 (2011), 113.

https://doi.org/10.1186/1756-3305-4-113 
[17] A. J. Turkson, Survival analysis modeling on level of exposure to the environment and health hazards, International Journal of Ecological Economics and Statistics 22 (2011), 33-49.

[18] R. E. Mbu, W. A. Takang, H. J. Fouedjio, F. Y. Fouelifack, F. N. Tumasang and R. Tonye, Clinical malaria among pregnant women on combined insecticide treated nets (ITNs) and intermittent preventive treatment (IPTp) with sulphadoxine-pyrimethamine in Yaounde, Cameroon, BMC Women's Health 14 (2014), 68. https://doi.org/10.1186/1472-6874-14-68

[19] D. G. Ayele, T. T. Zewotir and H. G. Mwambi, Prevalence and risk factors of malaria in Ethiopia, Malar. J. 11 (2012), 195. https://doi.org/10.1186/1475-2875-11-195

[20] E. S. Owusu Adjah and A. G. Panayiotou, Impact of malaria related messages on insecticide-treated net (ITN) use for malaria prevention in Ghana, Malar. J. 13 (2014), 123. https://doi.org/10.1186/1475-2875-13-123

[21] S. Singh and R. Singh, Awareness, ownership, and utilization of bed nets in rural areas of Aliero, Kebbi state, Nigeria, Global Journal of Biology Agriculture and Health Science 2 (2013), 125-129.

[22] A. Kilian, M. Boulay, H. Koenker and M. Lynch, How many mosquito nets are needed to achieve universal coverage? Recommendations for the quantification and allocation of long-lasting insecticidal nets for mass campaigns, Malar. J. 9 (2010), 330. https://doi.org/10.1186/1475-2875-9-330

[23] C. J. Mba and I. K. Aboh, Prevalence and management of malaria in Ghana: A case study of Volta region, African Population Studies 22(1) (2006), 138-165.

[24] S. Smith, Determining sample size: how to make sure you get the correct sample size, 2013. http://www.qualtrics.com/blog/determining-sample-size/

[25] K. D. Konlan, M. Japiong, K. D. Konlan, A. Afaya, S. M. Salia and J. M. Kombat, Utilization of Insecticide Treated Bed Nets (ITNs) among caregivers of children under five years in the Ho municipality, Interdisciplinary Perspectives on Infectious Diseases, 2019. https://doi.org/10.1155/2019/3693450

This is an open access article distributed under the terms of the Creative Commons Attribution License (http://creativecommons.org/licenses/by/4.0/), which permits unrestricted, use, distribution and reproduction in any medium, or format for any purpose, even commercially provided the work is properly cited. 